\title{
Über die funktionelle Schädigung der Nieren bei experimenteller Kieselgurgranulombildung des Organs.
}

\author{
Von \\ Dr. med. Masao Muto. \\ (武 藤 宗 雄) \\ (Aus der chinurgischen Universitätsklinik ron Prof. \\ Sh. Sugimura, Sendai.)
}

\section{Einleitung.}

Es ist seit langem bekaunte Tatsache, dass bei der Einspritzung von Kieselgur in das Gewebe sich ein Riesenzellengranulom an der Stelle der Injelition bildet. Auf diese Granulombildung machte Podwyssozki zuerst aufmerksam und studierte die Entstehung des Granuloms experimentell bei Meerschweinchen, indem er eine sterile Aufschwemmung fein zerriebener Kieselgur in die Bauchhöhle einspritzte. Die Zellwucherungen sollen nach dem Autor dabei hauptsächlich auf die mechanische Reizung von Kieselgur, teilweise aber auch auf ihre chemische Wirkung zurückzuführen sein.

Auch Deton stellte bei seiner Nachprüfung die von Podwyssozki gefundene Tatsache fest, fand aber, dass die Granulomknötchen nicht weiter wachsen können, wenn sie nach Ablauf eines gewissen Zeitraums eine bestimmte Grösse erreicht haben. Schirokogoroff machte auch Experimente bei Kaninchen und Hunden mit fein zerriebener Kieselgur und bemerkte an den dadurch erzeugten Granulomknötchen keine Tendenz zur Schrumpfung des Gewebes, wodurch der Prozess der Granulombildung deutlich vom Entzündungsprozess zu unterscheiden war.

Ich selbst konnte auch nach dem Vorgang der genannten Autoren durch Injektion fein zerriebener Kieselgur in steriler Kochsalzlösung in das Nierenparenchym bei Kaninchen Riesenzellengranulome in ihm erzeugen und dadurch Einengung des Nierenparenchyms als Folge der Granulombildung an verschiedenen Stellen des Nierenparenchy ms herbeiführen. Die dadurch hervorgerufene Beeinträchtigung der Funktion der Nieren 
wurde dabei geprüft. Meine Versuchsfülle zur Prüfung der Nierenfunktion bei ihrer Schädigung durch Kieselgurgranulombildung in der Niere bezichen sich auf 31 Kaninchen. Im Folgenden berichte ich kurz die Resultate meiner Experimente.

\section{Meine Versuchsanordnung.}

Es wurden bei meinen Experimenten im ganzen 41 erwachsene männliche Kaninchen verwendet. Bei den Tieren habe ich jedesmal ohne Narkose und auf lumbalem Wege eine oder beide Nieren freigelegt und mit feiner Nadel jedesmal für eine Niere zumeist 1,0, selten 1,5 ccm einer Aufschwemmung von im Mörser fein zerriebener Kieselgur in physiologischer Kochsalzlösung in mehreren Portionen in 2 bis 5 Stellen des Nierenparenchyms, und zwar in die Rindensubstanz tief bis ins Markgewebe injiziert.

Vor der Einspritzung der Kieselguraufschwemmung wurden bei den Tieren Funktionsprüfungen der Nieren angestellt. Eine gewisse Zeit nach wiederholten Funktionsprüfungen der Nieren wurde das Tier durch Nackenschlag getötet und seziert. Als Methoden der Nierenfunktionsprüfung wurden bei den Versuchsfällen die Gefrierpunktbestimmung sowie die Harnstoffbestimmung des Harns und des Blutes und vor allem auch die Phenolsulfophthaleinprobe angewandt. Bei der Selktion der Tiere wurden ausser den Nieren auch andere lebenswichtige Organe einer genauen makroskopischen Untersuchung unterzogen. Ausserdem wurden die Nieren, vor allem auch die erkrankte mikroskopisch untersucht. Dabei bediente ich mich hauptsächlich der Ḧ̈matoxylin-Eosinfärbung und der Van Giesonschen Fürbung des Paraffinschnittpräparates.

Von meinen sämtlichen 41 Tieren konnte ich bei 31 Granulombildung in der Niere feststellen. Bei 10 Versuchsfällen, wo zu fein zerriebene Kieselgur injiziert worden war, blieb Granulombildung vollständig aus, was auch aus der histologischen Untersuchung nachgewiesen wurde. Die übrigen 31 Tiere mit Granulombildung lassen sich in 2 Versuchsreihen cinteilen. Bei der Versuchsreihe A wurden nämlich bei 19 Kaninchen Granulome in der einen (linken) Niere erzeugt, während die andere Niere ganz unberührt gelassen wurde. Zwei bis secks, seltener acht Wochen nach der Kieselgurinjektion wurden die Tiere getötet und seziert. In der Versuchsreibe $\mathrm{B}$ wurden bei 12 Kaninchen die beiden Nieren operativ freigelegt, und die Kieselgurauf'schwemmung in ihr Parenchym injiziert. Die injizierte Menge der Kieselguraufschwemmung betrug 1,0 cem für jede ein- 
zelne Niere. Die Menge wurde dabei, wie oben erwähnt, in 4 oder 5 Portionen geteilt, in mehrere Stellen des Parenchyms eingespritzt. Nach Ablauf von 2 bis 8 Wochen nach der Injektion wurden die Tiere wie bei der Versuchsreihe A getötet und seziert. Bei allen diesen Tieren der beiden Versuchsreihen konnte man den Stellen der Injektion entsprechend mehr oder minder deutliche Granulombildung im Nierenparenchym feststellen.

\section{Ergebnisse meiner Versuche.}

Die Resultate aus meinen Versuchsreihen A und B werde ich übersichtshalber in folgenden Skizzen meiner Protokolle und in zwei Tabellen (siehe Tabelle I und II) wiedergeben.

\section{Versuchsreihe A.}

Versuch 1. Kaninchen, braun, $2,3 \mathrm{~kg}$. Injektionen an 3 verschiedenen Stellen der Niere vorgenommen, 2 Wochen nach der Injektion getötet. Sektionsbefund: Die linke Niere wiegt $7,2 \mathrm{~g}$. Zwei punktförmige und eine erbsengrosse Knötchenbildung in ihrem Parenchym. Mikroskopisch typisches Bild des Riesenzellengranuloms.

Versuch 2. Kaninchen, weiss, $2,0 \mathrm{~kg}$. Injektionen an 4 Stellen der linken Niere rorgenommen. Tötung nach 2 Wochen. Sektionsbefund: Die linke Niere $7,2 \mathrm{~g}$ schwer. Makroskopisch 4 reiskorn- bis erbsengrosse Knötchenbildung im Parenchym. Mikroskopisch wie bei Versuch 1.

Versuch 3. Kaninchen, weiss, $2,0 \mathrm{~kg}$. Injektionen an 4 Stellen der linken Niere. Tötung nach 2 Wochen. Sektionsbefund: Die linke Niere 6,5 g. Makroskopisch 4 feine, stäbchenförmige Herde neben einer bohnengrossen subkapsulären Knötchenbildung im $\mathrm{Pa}^{-}$ renchym festgestellt. Mikroskopisch wie bei Versuch 1.

Versuch 4. Kaninchen, weiss, $2,3 \mathrm{~kg}$. Injektionen an 5 Stellen der linken Niere vorgenommen. Tötung nach 3 Wochen. Sektionsbefund: Die linke Niere $7,5 \mathrm{~g}$ schwer. Ein punktförmiges und 4 reiskorn- bis erbsengrosse Knötchen in Parenchym konstatiert. Mikroskopisch wie bei Versuch 1.

Versuch 5. Kaninchen, weiss, $2,1 \mathrm{~kg}$. Injektionen an 4 Stellen der linken Niere rorgenommen. Tötung nach 3 Wochen. Sektionsbefund: Die linke Niere $6,2 \mathrm{~g}$ schwer. Makroskopisch ein feiner, stïbchenförmiger Herd und eine bohnengrosse zystische Neubildung im Parenchym konstatiert. Mikroskopisch zeigen die beiden Tumoren das typische Bild des Riesenzellengranuloms, und der zystische Tumor ist nichts anderes als ein Granulomknötchen, das im Zentrum Kieselgurbrei entb:̈lt.

Versuch 6. Kaninchen, weiss, $2,1 \mathrm{~kg}$. Injektionen in 4 rerschiedene Stellen des linken vorgenommen. Tötung nach 3 Wochen. Sektionsbefund: Die linke Niere $7,5 \mathrm{~g}$ schwer. Makroskopisch Bildung 4 reiskorngrosser Knötchen in ihrem Parenchym konstatiert. Mikroskopisch typisches Bild des Riesenzellengranuloms.

Versuch 7. Kaninchen, weiss, $2,8 \mathrm{~kg}$. Injektionen in 4 Stellen der linken Niere rorgenommen. Tötung nach 4 Wochen. Sektionsbefund: Die linke Niere $13,5 \mathrm{~g}$ schwer. Makroskopisch 2 reiskorngrosse Knötchen und 2 feine streifenartige Herde in Parenchym konstatiert. Mikroskopisch wie beim rorigen Versuch. 
Versuch 8. Kaninchen, braun, $2,7 \mathrm{~kg}$. Injektionen in 4 Stellen der Niere rorgenommen. Tötung nach 4 Wochen. Sektionsbefund: Die linke Niere $9,5 \mathrm{~g}$ schwer. Makroskopisch 2 punlitförmige und 2 erbsengrosse Knötchen im Parenchym konstatiert. Mikroskopisch wie bei Versuch 6 .

Versuch 9. Kaninchen, gefleckt, $2,1 \mathrm{~kg}$. Injektionen in 3 Stellen der linken Niere rorgenommen. Tötung nach 4 Wochen. Sektionsbefund: Die linke Niere $8,0 \mathrm{~g}$ schwer. Makroskopisch Bildung 2 reiskorngrosser Knötchen im Parenchym und 2 erbsen- resp. bohnengrosser, subliapsulär gelegener Knötchen festgestellt. Mikroskopisch wie bei Versuch 6.

Versuch 10. Kaninchen, braun, $2,6 \mathrm{~kg}$. Injektionen in 4 Stellen der linken Niere rorgenommen. Tötung nach 5 Wochen. Sektionsbefund: Die linke Niere $9,3 \mathrm{~g}$ schwer. Makroskopisch Bildung 2 erbsengroseer und 2 teiner, stäbchenförmiger Herde im Parenchym konstatiert. MTikroskopisch wie bei Versuch 6 .

Versuch 11. Kaninchen, braun, $2,5 \mathrm{~kg}$. Injektionen in 4 Stellen der linken Niere rorgenommen. Tötung nach 5 Wochen. Sektionsbefund: Die linke Niere $8,5 \mathrm{~g}$ schwer. Makroskopisch eine bohnengrosse Knötchenbildung im Nierenbecken und Bildung 4 radiür von der Nierenoberflüche nach dem Knötchen des Nierenbeckens verlaufender, nadelförmiger Granulomstreifen in Parenchym konstatiert. Das Nierenbeckenlumen noch erhalten. Mikroskopisch typisches Bild des Riesenzellengranuloms.

Versuch 12. Kaninchen, braun, $2,1 \mathrm{~kg}$. Injektionen in 4 Stellen der linken Niere rorgenowmen. Tötung nach 5 Wochen. Sektionsbefund: Die linke Niere $6,3 \mathrm{~g}$ schwer. Makroskopisch 3 reiskorngrosse und ein erbsengrosses Knötchen in Parenchym festgestellt. Mikroskopisch typisches Bild des Riesenzellengranuloms.

Versuch 13. Kaninchen, weiss, $2,2 \mathrm{~kg}$. Injektionen in 4 Stellen der linken Niere ausgefüht. Tötung nach 6 Wochen. Sektionsbefund: Die linke Niere $9,0 \mathrm{~g}$ schwer. Makroskopisch ein bo hnengrosses Knötchen mit Kieselgurbrei in seinem Zentrum im Parenchym festgestellt. Makroskopisch wie bei Versuch 12.

Versuch 14. Kaninchen, gefleckt, 2,2 kg. Injektionen in 5 Stellen der linken Niere rorgenommen. Tötung nach 6 Wochen. Sektionsbefund: Die linke Niere $7,0 \mathrm{~g}$ schwer. Makroskopisch 2 reiskorn- und ein erbsengrosses Knötchen im Parenchym konstatiert. Mikroskopisch wie beim Versuch 12.

Versuch 15. Kaninchen, braun, 2,0 kg. Injektionen an 4 Stellen der linken Niere ausgefuhrt. Tötung nach 6 Wochen. Sektionsbefund: Die linke Niere $6,5 \mathrm{~g}$ schwer. Makroskopisch ein reiskorngrosses und 2 erbsengrosse Knötchen im Parenchym konstatiert. Mikroskopisch wie bei Versuch 12.

Versuch 16. Kaninchen, weiss, $2,0 \mathrm{~kg}$. Injektionen in 4 Stellen der linken Niere vorgenommen. Tötung nach 8 Wochen. Sektionsbefund: Die linke Niere $6,5 \mathrm{~g}$ schwer. Makroskopisch 2 reiskorngrosse Knötchen im Parenchym konstatiert. Mikroskopisch wie bei Versuch 12.

Versuch 17. Kaninchen, weiss, $2,0 \mathrm{~kg}$. Injektionen an 4 Stellen der linken Niere ausgefïhrt. Tötung nach $\$$ Wochen. Selitionsbefund: Die linke Niere $7,5 \mathrm{~g}$ schwer. Makroskopisch ein erbsengrosses und 2 punktförmige Knötchen neben einew streifenförmigen Herd im Parenchyn festgestellt. Mikroskopisch wie bei Versuch 12.

Versuch 18. Kaninchen, weiss, $1,9 \mathrm{~kg}$. Injektionen in 4 Stellen der linken Niere vorgenommen. Tötung nach 8 Wochen. Sektionsbefund: Die linke Niere 7,0 g schwer. Makroskopisch 3 erbsengrosse Neubildungen im Parenchym. Mikroskopisch wie bei Versuch 12.

Versuch 19. Kaninchen, weiss, $2,3 \mathrm{~kg}$. Injektionen in 5 Stellen der linken Niere vorgenommen. Tötung nach 8 Wochen. Sektionsbefund: Die linke Niere $6,5 \mathrm{~g}$ schwer. Makroskopisch 3 erbsengrosse und ein miliumgrosses Knötchen im Parenchym konstatiert. Mikroskopisch wie bei Versuch 12. 
Tabelle I.

Versuchsreihe A.

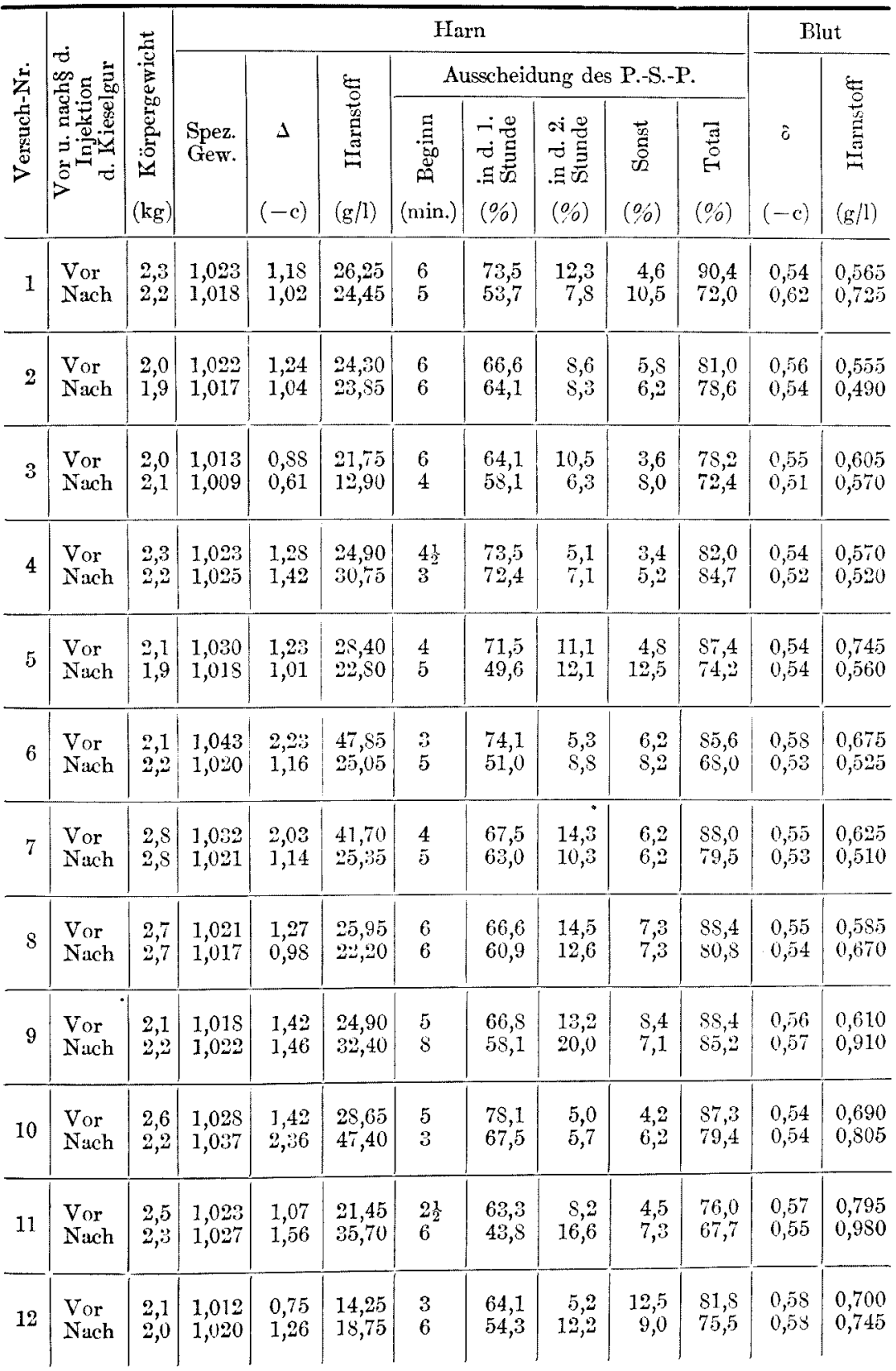




\begin{tabular}{|c|c|c|c|c|c|c|c|c|c|c|c|c|}
\hline \multirow{3}{*}{$\frac{\dot{H}}{\stackrel{1}{\leftrightarrows}}$} & \multirow{3}{*}{ 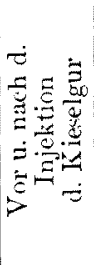 } & \multirow{3}{*}{ 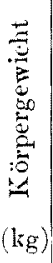 } & \multicolumn{8}{|c|}{ Harn } & \multicolumn{2}{|c|}{ Biut } \\
\hline & & & \multirow[b]{2}{*}{$\begin{array}{l}\text { Spez. } \\
\text { Gew. }\end{array}$} & \multirow[b]{2}{*}{$\begin{array}{c}\Delta \\
(-c)\end{array}$} & \multirow{2}{*}{ 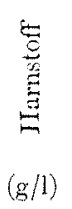 } & \multicolumn{5}{|c|}{ Ausscheidung des P.-S.-P. } & \multirow[b]{2}{*}{$\begin{array}{c}\delta \\
(-c)\end{array}$} & \multirow[b]{2}{*}{ 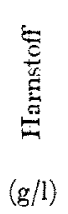 } \\
\hline & & & & & & 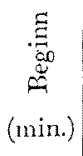 & 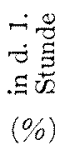 & 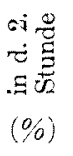 & 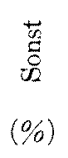 & $\begin{array}{l}\overrightarrow{\vec{F}} \\
\stackrel{0}{0} \\
(\%)\end{array}$ & & \\
\hline 13 & $\begin{array}{l}\text { Vor } \\
\text { Nach }\end{array}$ & $\begin{array}{l}2,2 \\
2,3\end{array}$ & $\begin{array}{l}1,038 \\
1,018\end{array}$ & $\begin{array}{l}2,68 \\
1,11\end{array}$ & $\begin{array}{l}49,95 \\
22,50\end{array}$ & $\begin{array}{l}5 \\
5\end{array}$ & $\begin{array}{l}79,3 \\
54,3\end{array}$ & $\begin{array}{r}5,8 \\
10,0\end{array}$ & $\begin{array}{l}4,7 \\
5,2\end{array}$ & $\begin{array}{l}89,8 \\
69,5\end{array}$ & $\begin{array}{l}0,56 \\
0,52\end{array}$ & $\begin{array}{l}0,825 \\
0,610\end{array}$ \\
\hline 14 & $\begin{array}{l}\text { Vor } \\
\text { Nach }\end{array}$ & $\begin{array}{l}2,2 \\
2,3 \\
2,3\end{array}$ & $\begin{array}{l}1,016 \\
1,016\end{array}$ & $\begin{array}{l}1,09 \\
1,02\end{array}$ & $\begin{array}{l}24,60 \\
18,00\end{array}$ & $\begin{array}{l}5 \\
6\end{array}$ & $\begin{array}{l}75,7 \\
52,0\end{array}$ & $\begin{array}{r}5,5 \\
12,5\end{array}$ & $\begin{array}{l}5,0 \\
7,1\end{array}$ & $\begin{array}{l}86,2 \\
71,6\end{array}$ & $\begin{array}{l}0,56 \\
0,53\end{array}$ & $\begin{array}{l}0,805 \\
0,635\end{array}$ \\
\hline 10 & $\begin{array}{l}\text { Vor } \\
\text { Nach }\end{array}$ & $\begin{array}{l}2,0 \\
1,8\end{array}$ & $\stackrel{1,017}{-}$ & $\begin{array}{l}1,11 \\
1,56\end{array}$ & $\begin{array}{l}21,90 \\
38,70\end{array}$ & $\begin{array}{l}3 \\
7\end{array}$ & $\begin{array}{l}66,6 \\
59,5\end{array}$ & $\begin{array}{r}4,3 \\
13,3\end{array}$ & $\begin{array}{l}6,2 \\
6,2\end{array}$ & $\begin{array}{l}77,1 \\
79,0\end{array}$ & $\begin{array}{l}0,54 \\
0,59\end{array}$ & $\begin{array}{l}0,670 \\
0,885\end{array}$ \\
\hline 16 & $\begin{array}{l}\text { Vor } \\
\text { Nach }\end{array}$ & $\begin{array}{l}2,1 \\
2,1\end{array}$ & $\begin{array}{l}1,024 \\
1,023\end{array}$ & $\begin{array}{l}1,16 \\
1,26\end{array}$ & $\begin{array}{l}35,40 \\
29,70\end{array}$ & $\frac{6}{7}$ & $\begin{array}{l}66,6 \\
57,9\end{array}$ & $\begin{array}{r}9,1 \\
11,4\end{array}$ & $\begin{array}{l}10,1 \\
12,5\end{array}$ & $\begin{array}{l}85,8 \\
81,8\end{array}$ & $\begin{array}{l}0,56 \\
0,56\end{array}$ & $\begin{array}{l}0,860 \\
0,855\end{array}$ \\
\hline 17 & $\begin{array}{l}\text { Vor } \\
\text { Nach }\end{array}$ & $\begin{array}{l}2,0 \\
2,2\end{array}$ & $\begin{array}{l}1,023 \\
1,019\end{array}$ & $\begin{array}{l}1,38 \\
1,28\end{array}$ & $\begin{array}{l}30,30 \\
27,45\end{array}$ & $\begin{array}{l}5 \\
4\end{array}$ & $\begin{array}{l}74,7 \\
61,8\end{array}$ & $\begin{array}{r}7,2 \\
20,4\end{array}$ & $\begin{array}{l}5,4 \\
7,0\end{array}$ & $\begin{array}{l}87,3 \\
89,2\end{array}$ & $\begin{array}{l}0,56 \\
0,52\end{array}$ & $\begin{array}{l}0,840 \\
0,505\end{array}$ \\
\hline 18 & $\begin{array}{l}\text { Vor } \\
\text { Nach }\end{array}$ & $\begin{array}{l}1,9 \\
2,0\end{array}$ & $\begin{array}{l}1,019 \\
1,022\end{array}$ & $\begin{array}{l}1,02 \\
1,43\end{array}$ & $\begin{array}{l}23,85 \\
34,20\end{array}$ & $\begin{array}{l}3 \\
5\end{array}$ & $\begin{array}{l}75,9 \\
51,9\end{array}$ & $\begin{array}{r}8,7 \\
18,5\end{array}$ & $\begin{array}{r}6,2 \\
12,7\end{array}$ & $\begin{array}{l}90,8 \\
83,1\end{array}$ & $\begin{array}{l}0,59 \\
0,53\end{array}$ & $\begin{array}{l}0,725 \\
0,525\end{array}$ \\
\hline 19 & $\begin{array}{l}\text { Vor } \\
\text { Nach }\end{array}$ & $\begin{array}{l}2,8 \\
2,1\end{array}$ & $\begin{array}{l}1,034 \\
1,016\end{array}$ & $\begin{array}{l}2,09 \\
0,81\end{array}$ & $\begin{array}{l}38,10 \\
16,05\end{array}$ & $\begin{array}{l}7 \\
7\end{array}$ & $\begin{array}{l}69,8 \\
58,5\end{array}$ & $\begin{array}{r}8,7 \\
13,5\end{array}$ & $\begin{array}{l}6,1 \\
6,2\end{array}$ & $\begin{array}{l}84,6 \\
78,2\end{array}$ & $\begin{array}{l}0,54 \\
0,52\end{array}$ & $\begin{array}{l}0,505 \\
0,885\end{array}$ \\
\hline
\end{tabular}

$\$$ Das Körpergewicht, das spezifische Gewicht des Harns, und die Gefrierpunkt- und Iramstoffbestimmung des IIarns und Blutes 2 Tage, die P.-S.-P.-Probe einen Tag vor der Tötung des Tiers vorgenommen.

\section{Versuchsreihe B.}

Versuch 20. Kaninchen, weiss, $2,3 \mathrm{~kg}$. Injektionen in je 4 Stellen beider Nieren vorgenommen. Tötung nach 2 Wochen. Sektionsbefund: Die rechte Niere $7,5 \mathrm{~g}$, die linke $8,0 \mathrm{~g}$ schwer. Makroskopich ein erbsengrosses Knötchen und 3 streifenförmige Herde im Parenchym der rechten, 2 punktförmige und 2 erbsengrosse Knötchen in den der linken Niere konstatiert. Mikroskopisch typisches Bild des Riesenzellengranuloms.

Versuch 21. Kaninchen, gefleckt, 2,3 lig. Injektionen in je 4 Stellen beider Nieren vorgenommen. Tötung nach \& Wochen. Sektionsbefund: Die rechte Niere $9,0 \mathrm{~g}$, die linke $10,0 \mathrm{~g}$ schwer. Makroskopisch 3 erbsengrosse Knötchen und ein nadelförmiger Herd im Parenchym der rechten und 2 erbsengrosse und 2 reiskorngrosse Knötchen in dem der linken Niere festgestellt. Mikroskopisch wie bei Versuch 20.

Versuch 22. Kaninchen, weiss, $2,2 \mathrm{~kg}$. Injektionen in je 4 Stellen beider Nieren rorgenommen. Tötung nach 2 Wochen. Sektionsbefund: Die rechte Niere $7,5 \mathrm{~g}$, die linke 
7,4 g schwer. Makroskopisch 2 erbsengrosse Knötchen im Parenchym der rechten und 3 ebensolche in dem der linken Niere konstatiert. Mikroskopisch wie bei Versuch 20 .

Versuch 23. Kaninchen, schwarz, 2,1 kg. Injektionen an 4 Stellen der rechten und an 5 Stellen der linken Niere ausgeführt. Tötung nach 3 Wochen. Sektionsbefund: Die rechte Niere $\$, 2 \mathrm{~g}$, die linke $9,4 \mathrm{~g}$ schwer. Makroskopisch 2 punktförmige und 2 reiskorngrosse Knötchen im Parenchym der rechten und 4 erbsengrosse Knötchen in dem der linken Niere konstatiert. Mikroskopisch wie bei Versuch 20.

Versuch 24. Kaninchen, weiss, $2,1 \mathrm{~kg}$. Injektionen in 5 Stellen der rechten und in 4 Stellen der linken Niere vorgenommen. Tötung nach 3 Wochen. Sektionsbefund: Die rechte Niere $7, \mathrm{Sg}$, die linke $7,7 \mathrm{~g}$ schwer. Makroskopisch 3 reiskorngrosse und ein erbsengrosses Knötchen im Parenchym der rechten und 2 erbsengrosse und ein reiskorngrosses Knötchen in dem der linken Niere konstatiert. Mikroskopisch wie bei Versuch 20.

Versuch 25. Kaninchen, braun, $2,8 \mathrm{~kg}$. Injektionen in je $4 \mathrm{Stellen}$ beider $\mathrm{Nieren}$ ausgefuhrt. Tötung nach 3 Wochen. Sektionsbefund: Die rechte Niere $10,5 \mathrm{~g}$, die linke 10,3 g schwer. Makroskopisch 2 erbsengrosse Knötchen und 2 nadelförmige Herde im Parenchym der rechten und ein erbsengrosses und ein reiskorngrosses $K$ nötchen sowie ein streifenförmiger Herd und ein erbsengrosses subkapsuläres Knötchen in Parenchym der linken Niere konstatiert. Mikroskopisch wie bei Versuch 20.

Versuch 26. Kaninchen, weiss, 2,8 kg. Injektionen in je 4 Stellen beider Nieren vorgenommen. Tötung nach 4 Wochen. Sektionsbefund: Die rechte Niere $11,0 \mathrm{~g}$, die linke $10,0 \mathrm{~g}$ schwer. Makroskopisch 2 stäbchenförmige und ein erbsengrosses Knötchen im Parenchym der rechten und 2 miliumgrosse Knötchen und 2 streifenförmige Herde in dem der linken Niere nachgewiesen. Mikroskupisch wie bei Versuch 20.

Versuch 27. Kaninchen, weiss, $2,0 \mathrm{~kg}$. Injektionen in je 4 Stellen beider Nieren vorgenommen. Tötung nach 4 Wochen. Sektionsbefund: Die rechte Niere $9,0 \mathrm{~g}$, die linke $8,4 \mathrm{~g}$ schwer. Milkroskopisch mïssig breiter, von der Oberfläche bis zum Nierenbecken verlaufender, stübchenförmiger Herd neben einem punktförmigen Knötchen in Parenchym der rechten und 3 punktförmige Knötchen in dem der linken Niere festgestellt. Mikroskopisch das typische Bild des Riesenzellengranuloms.

Versuch 28 . Kaninchen, braun, $2,7 \mathrm{~kg}$. Injektionen in je 4 Stellen beider Nieren vorgenommen. Tötung nach 4 Wochen. Sektionsbefund: Die rechte Niere $10,5 \mathrm{~g}$, die linke $10,2 \mathrm{~g}$ schwer. Makroskopisch 2 reiskorngrosse Knötchen und 2 streifenförmige Herde im Parenchym der rechten und eine reiskorngrosse Knötchenbildung in dem der linken Niere festgestellt. Makroskopisch wie bei Versuch 27.

Versuch 29. Kaninchen, braun, 1,9 kg. Injektionen an je 4 Stellen beider Nieren ausgeführt. Tötung nach 8 Wochen. Sektionsbefund: Die rechte Niere $4,0 \mathrm{~g}$, die linke $4,0 \mathrm{~g}$ schwer. Makroskopich 3 reiskorngrose Knötchen im Parenchym beider Nieren festgestellt. Mikroskopisch wie bei Versuch 27.

Versuch 30 . Kaninchen, braun, $3,2 \mathrm{~kg}$. Injektionen in je 4 Stellen beider Nieren vorgenommen. Tötung nach $S$ Wochen. Sektionsbefund: Die rechte Niere $10,0 \mathrm{~g}$, die linke $8,2 \mathrm{~g}$ schwer. Makroskopisch 3 erbsengrosse Kuötchen in Parenchym der rechten und 3 reiskorngrosse Knötchen in dem der linken Niere nachgewiesen. Mikroskopisch dus typische Bild des Riesenzellengranuloms.

Versuch 31 . Kaninchen, weiss, $2,4 \mathrm{~kg}$. Injektionen an 4 Stellen der rechten Niere und an 3 Stellen der linken Niere ausgefuhrt. Tötung nach 8 Wochen. Sektionsbefund : Die rechte Niere $9,0 \mathrm{~g}$, die linke $8,0 \mathrm{~g}$ schwer. Nakroskopisch 2 reiskorngrosse und ein erbsengrosses Knötchen im Parenchym der rechten und ein reiskorngrosses und ein erbsengrosses Knötchen in dem der linken Niere festgestellt. Mikroskopisch wie bei Versuch 31. 
Tabelle II.

Versuchsreihe B.

\begin{tabular}{|c|c|c|c|c|c|c|c|c|c|c|c|c|}
\hline \multirow{3}{*}{$\frac{\stackrel{5}{Z}}{\frac{1}{5}}$} & \multirow{3}{*}{ 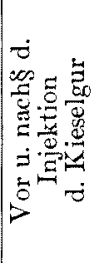 } & \multirow{3}{*}{ 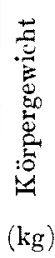 } & \multicolumn{8}{|c|}{ Harn } & \multicolumn{2}{|c|}{ Blut } \\
\hline & & & \multirow[b]{2}{*}{$\begin{array}{l}\text { Spez. } \\
\text { Gew. }\end{array}$} & \multirow[b]{2}{*}{$\begin{array}{c}\Delta \\
(-\mathrm{c})\end{array}$} & \multirow{2}{*}{ 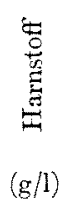 } & \multicolumn{5}{|c|}{ Ausscheidung des P.-S.-P. } & \multirow[b]{2}{*}{$\begin{array}{c}\hat{o} \\
(-c)\end{array}$} & \multirow{2}{*}{ 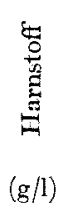 } \\
\hline & & & & & & 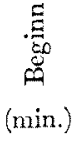 & 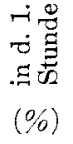 & 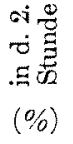 & 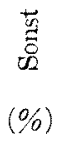 & $\begin{array}{l}\frac{E}{0} \\
E \\
(\%)\end{array}$ & & \\
\hline 20 & $\begin{array}{l}\text { Vor } \\
\text { Nach }\end{array}$ & $\begin{array}{l}2,3 \\
2,2\end{array}$ & $\begin{array}{l}1,017 \\
1,010\end{array}$ & $\begin{array}{l}0,87 \\
0,60\end{array}$ & $\begin{array}{l}14,55 \\
14,55\end{array}$ & $\begin{array}{l}5 \\
6\end{array}$ & $\begin{array}{l}64,1 \\
55,5\end{array}$ & $\begin{array}{r}5,8 \\
13,1\end{array}$ & $\begin{array}{r}3,3 \\
10,0\end{array}$ & $\begin{array}{l}73,2 \\
78,6\end{array}$ & $\begin{array}{l}0,56 \\
0,52\end{array}$ & $\begin{array}{l}0,625 \\
0,495\end{array}$ \\
\hline 21 & $\begin{array}{l}\text { Vor } \\
\text { Nach }\end{array}$ & $\begin{array}{l}2,3 \\
2,0\end{array}$ & $\begin{array}{l}1,038 \\
1,012\end{array}$ & $\begin{array}{l}2,14 \\
0,67\end{array}$ & $\begin{array}{l}41,40 \\
16,05\end{array}$ & $\begin{array}{l}5 \\
6\end{array}$ & $\begin{array}{l}76,9 \\
67,5\end{array}$ & $\begin{array}{l}4,7 \\
7,1\end{array}$ & $\begin{array}{l}3,5 \\
1,5\end{array}$ & $\begin{array}{l}\$ 5,1 \\
76,1\end{array}$ & $\begin{array}{l}0,55 \\
0,51\end{array}$ & $\begin{array}{l}0,610 \\
0,015\end{array}$ \\
\hline 22 & $\begin{array}{l}\text { Vor } \\
\text { Nach }\end{array}$ & $\begin{array}{l}2,2 \\
2,1\end{array}$ & $\begin{array}{l}1,014 \\
1,015\end{array}$ & $\begin{array}{l}3,81 \\
0,90\end{array}$ & $\begin{array}{l}37,05 \\
19,35\end{array}$ & $\begin{array}{l}5 \\
6\end{array}$ & $\begin{array}{l}67,5 \\
60,2\end{array}$ & $\begin{array}{r}8,6 \\
13,1\end{array}$ & $\begin{array}{l}4,2 \\
7,1\end{array}$ & $\begin{array}{l}\mathrm{S} 0,3 \\
\mathrm{~s} 0,4\end{array}$ & $\begin{array}{l}0,54 \\
0,54\end{array}$ & $\begin{array}{l}0,545 \\
0,505\end{array}$ \\
\hline 23 & $\begin{array}{l}\text { Vor } \\
\text { Nach }\end{array}$ & $\begin{array}{l}2,1 \\
2,0\end{array}$ & $\begin{array}{l}1,025 \\
1,023\end{array}$ & $\begin{array}{l}1,41 \\
1,11\end{array}$ & $\begin{array}{l}27,90 \\
24,30\end{array}$ & $\begin{array}{l}6 \\
5\end{array}$ & $\begin{array}{l}67,5 \\
59,5\end{array}$ & $\begin{array}{l}9,9 \\
5,5\end{array}$ & $\begin{array}{l}6,2 \\
6,3\end{array}$ & $\begin{array}{l}83,6 \\
71,3\end{array}$ & $\begin{array}{l}0,54 \\
0,55\end{array}$ & $\begin{array}{l}0,555 \\
0,615\end{array}$ \\
\hline 24 & $\begin{array}{l}\text { Vor } \\
\text { Nach }\end{array}$ & $\begin{array}{l}2,1 \\
2,1\end{array}$ & $\frac{1,013}{-}$ & $\begin{array}{l}0,90 \\
1,50\end{array}$ & $\begin{array}{l}19,80 \\
34,20\end{array}$ & $\begin{array}{l}4 \\
7\end{array}$ & $\begin{array}{l}74,6 \\
46,5\end{array}$ & $\begin{array}{r}7,7 \\
10,4\end{array}$ & $\begin{array}{l}4,2 \\
7,5\end{array}$ & $\begin{array}{l}86,5 \\
64,4\end{array}$ & $\begin{array}{l}0,58 \\
0,54\end{array}$ & $\begin{array}{l}0,502 \\
0,595\end{array}$ \\
\hline 25 & $\begin{array}{l}\text { Vor } \\
\text { Nach }\end{array}$ & $\begin{array}{l}2,8 \\
2,6\end{array}$ & $\begin{array}{l}1,024 \\
1,019\end{array}$ & $\begin{array}{l}1,45 \\
1,16\end{array}$ & $\begin{array}{l}22,80 \\
28,50\end{array}$ & $\begin{array}{l}4 \\
6\end{array}$ & $\begin{array}{l}79,3 \\
54,9\end{array}$ & $\begin{array}{l}5,0 \\
9,6\end{array}$ & $\begin{array}{l}8,6 \\
8,5\end{array}$ & $\begin{array}{l}87,9 \\
73,0\end{array}$ & $\begin{array}{l}0,52 \\
0,52\end{array}$ & $\begin{array}{l}0,485 \\
0,465\end{array}$ \\
\hline 26 & $\begin{array}{l}\text { Vor } \\
\text { Nach }\end{array}$ & $\begin{array}{l}2,8 \\
2,7\end{array}$ & $\begin{array}{l}1,018 \\
1,014\end{array}$ & $\begin{array}{l}1,08 \\
0,70\end{array}$ & $\begin{array}{l}21,30 \\
14,40\end{array}$ & $\begin{array}{l}4 \\
6\end{array}$ & $\begin{array}{l}65,0 \\
66,6\end{array}$ & $\begin{array}{r}9,2 \\
11,9\end{array}$ & $\begin{array}{l}5,7 \\
8,6\end{array}$ & $\begin{array}{l}79,9 \\
87,1\end{array}$ & $\begin{array}{l}0,53 \\
0,59\end{array}$ & $\begin{array}{l}0,515 \\
0,480\end{array}$ \\
\hline 27 & $\begin{array}{l}\text { Vor } \\
\text { Nach }\end{array}$ & $\begin{array}{l}2,0 \\
2,1\end{array}$ & $\begin{array}{l}1,016 \\
1,016\end{array}$ & $\begin{array}{l}0,86 \\
0,76\end{array}$ & $\begin{array}{l}18,75 \\
19,35\end{array}$ & $\begin{array}{l}5 \\
7\end{array}$ & $\begin{array}{l}74,4 \\
60,4\end{array}$ & $\begin{array}{r}7,9 \\
12,5\end{array}$ & $\begin{array}{r}7,1 \\
12,5\end{array}$ & $\begin{array}{l}89,4 \\
85,4\end{array}$ & $\begin{array}{l}0,52 \\
0,56\end{array}$ & $\begin{array}{l}0,505 \\
0,585\end{array}$ \\
\hline 28 & $\begin{array}{l}\text { Vor } \\
\text { Nach }\end{array}$ & $\begin{array}{l}2,7 \\
2,7\end{array}$ & $\begin{array}{l}1,019 \\
1,025\end{array}$ & $\begin{array}{l}1,12 \\
1,21\end{array}$ & $\begin{array}{l}24,15 \\
29,35\end{array}$ & $\begin{array}{l}32 \\
4\end{array}$ & $\begin{array}{l}78,1 \\
65,7\end{array}$ & $\begin{array}{r}10,4 \\
5,5\end{array}$ & $\begin{array}{l}5,5 \\
5,0\end{array}$ & $\begin{array}{l}94,0 \\
76,2\end{array}$ & $\begin{array}{l}0,52 \\
0,53\end{array}$ & $\begin{array}{l}0,545 \\
0,515\end{array}$ \\
\hline 29 & $\begin{array}{l}\text { Vor } \\
\text { Nach }\end{array}$ & $\begin{array}{l}1,8 \\
1,7\end{array}$ & $\begin{array}{l}1,018 \\
1,025\end{array}$ & $\begin{array}{l}1,34 \\
1,71\end{array}$ & $\begin{array}{l}27,00 \\
35,55\end{array}$ & $\begin{array}{l}5 \\
7\end{array}$ & $\begin{array}{l}68,2 \\
50,5\end{array}$ & $\begin{array}{l}10,1 \\
21,5\end{array}$ & $\begin{array}{r}4,0 \\
10,0\end{array}$ & $\begin{array}{l}82,3 \\
82,0\end{array}$ & $\begin{array}{l}0,58 \\
0,55\end{array}$ & $\begin{array}{l}0,780 \\
0,655\end{array}$ \\
\hline 30 & $\begin{array}{l}\text { Yor } \\
\text { Nach }\end{array}$ & $\begin{array}{l}3,3 \\
2,9\end{array}$ & $\begin{array}{l}1,024 \\
1,018\end{array}$ & $\begin{array}{l}1,60 \\
1,08\end{array}$ & $\begin{array}{l}22,05 \\
25,25\end{array}$ & $\begin{array}{l}4 \\
5\end{array}$ & $\begin{array}{l}76,8 \\
64,1\end{array}$ & $\begin{array}{l}8,6 \\
8,3\end{array}$ & $\begin{array}{l}5,0 \\
6,6\end{array}$ & $\begin{array}{l}90,4 \\
79,0\end{array}$ & $\begin{array}{l}0,55 \\
0,53\end{array}$ & $\begin{array}{l}0,560 \\
0,550\end{array}$ \\
\hline 3 & $\begin{array}{l}\text { Vor } \\
\text { Nach }\end{array}$ & $\begin{array}{l}2,4 \\
2,3\end{array}$ & $\begin{array}{c}1,018 \\
-\end{array}$ & $\begin{array}{l}0,90 \\
1,78\end{array}$ & $\begin{array}{l}15,45 \\
39,05\end{array}$ & $\begin{array}{l}3 \\
5\end{array}$ & $\begin{array}{l}71,4 \\
66,6\end{array}$ & $\begin{array}{l}7,3 \\
7,1\end{array}$ & $\begin{array}{l}5,8 \\
5,7\end{array}$ & $\begin{array}{l}84,5 \\
79,4\end{array}$ & $\begin{array}{l}0,55 \\
0,57\end{array}$ & $\begin{array}{l}0,635 \\
0,645\end{array}$ \\
\hline
\end{tabular}

$\$$ Siehe die Bemerkung auf der Seite 520 . 


\section{Zusammenfassende Betrachtungen.}

Ich konnte, wie oben erwähnt, bei allen Tieren meiner Versuchsreihen A und B mehr oder minder grosse Riesenzellengranulome an den Injektionsstellen des Nierenparenchyms erzeugen. Diese Gebilde kamen dabei makroskopisch öfters als Knötchen, manchmal auch als streifen- oder nadelförmige Herde vor und zeigten mikroskopisch das typische Bild der sogenannten Kieselgurriesenzellengranulome.

In allen diesen Granulomen waren histologisch, wie schon Podwy ssozki, Deton und Schirokogoroff u. a. bemerkten, recht zahlreiche, mehr oder minder grosse Riesenzellen und wenige junge Zellen nebeu spürlichem jungen Bindegewebe zu kostatieren. Fast alle Riesenzellen enthielten kleinste Kieselnadeln und -panzer in ihren Zentren, und ihre zahlreichen hyperchromatischen Kerne waren hauptsächlich in der Peripherie des Zelleibs, zuweilen aber zerstreut darin gelegen. Üfters sah man mehrere grosse, mit zahlreichen Kernen versehene Riesenzellen, die rings um einen Haufen kleinster Kieselnadeln und -panzer gelagert waren und manchmal miteinander zu einer enorm grossen Riesenzelle konfluierten. Die Granulome und zwar die älteren Datums wiesen recht zahlreiche Riesenzellen von verschiedener Grösse und Form auf, so dass sie unter dem Mikroskop aus lauter Riesenzellen zu bestehen schienen. Auch sah man mitunter Rundzellenanhüufungen in und neben dem Granulom und kleinere oder grössere Nekroseherde im Zentrum des Granulons, wo sich neben den Kieselkörperchen auch zerfallene, teilweise homogenisierte Gewebszellen fanden. Von den Glomeruli und Harnkanälchen zeigte sich keine Spur mehr im Granulomgewebe. Dieses wies ausser den Riesenzellen manchmal auch spärlich junges Bindegewebe auf, das in dünnen Streifen zwischen den Riesenzellen lag und nicht selten mehrere kleine, stark gefüllte Kapillaren in sich hatte.

Das oben erwähnte Riesenzellengranulom zeigte ferner eine mehr oder weniger deutlich entwickelte, kapselähnliche Bindegewebswucherung zwischen ihm und dem ihm angrenzenden Nierenparenchym. Das letztere war auch oft in direkter Nähe einer Granulomkapsel besonders verïndert. Das interstitielle Bindegewebe wurde dort mehr oder minder stark vermehrt vorgefunden und die Blutgef ïsse, und zwar Kapillaren, fast überall strotzend gefüllt. Die Glomeruli waren da stark an Zahl vermindert und zeigten oft deutliche Erweiterung des Kapselraumes. Die Harnkanälchen waren auch vermindert, zeigten auch öfters an etwas entfernteren Stellen vom Granulomgewebe dentliche Erweiterung der Lumen mit hyalinem Zylinder darin. In einigen Versuchsfällen kam Erweiterung des Bow- 
manschen Kapselraumes und der Harnkanälchen mit oder ohne Zylinderbildung in relativ grosser Ausdehnung in der etwas vom Granulom entfernten, subkortikal gelegenen Rindenpartie vor.

Alle histologischen Bilder der Kieselgurgranulome bei meinen Versuchsfällen entsprachen im grossen und ganzen den Beschreibungen der oben genannten Autoren. Dabei muss aber besonders hervorgehoben werden, dass die Glomeruli und die Harnkanälchen teils bei der Injektion der Kieselguraufschwenmung selbst, teils und hauptsächlich aber durch Zellwucherungen infolge der mechanischen Wirkungen der Kieselgur an der Stelle der Injektion im Nierenparenchy m fast ganz vernichtet worden waren, und dass in dem dem Granulom benachbarten Bezirk des Nierenparenchyms auch Veränderungen der Glomeruli und Marnkanälchen gefunden wurden. Auch muss beachtet werden, dass die Kieselgurgranulome nicht mehr weiter wachsen können, wenn sie eine gewisse Grösse erreicht haben, dann aber, wie schon Schirokogoroff bemerkte, fast keine Tendenz zur Scbrumpfung zeigen. Die Veründerungen an den Glomeruli und Harnkanälchen waren also bei meinen Versuchsfällen in den älteren und grösseren Knötchen verhältnismässig deutlich ausgeprägt.

Was nun die Funktionsprüfungen der anatomisch auf solche Weise geschädigten Niere betrifft, so konnte ich bei den Fällen der Versuchsreihe A (siehe Tabelle I), wo es sich um Granulombildung in der einen Niere handelte, fast keine ausgeprïgte Funktionsstörung der Nieren nachweisen. Dies lïsst sich auch teilweise aus der Möglichkeit einer Kompensation durch die andere Niere leicht verstehen. Bei den Fällen dieser Versuchsreihe waren nämlich im spezifischen Gewicht, Gefrierpunkt und Harnstoffgehalt des Harns sowie im Gefrierpunkt und Harnstoffgehalt des Blutes keine Besonderheiten hervorzuheben.

Die Phthaleinausscheidung war aber bei den Fällen der Versuchsreihe A (siehe Tabelle I) im grossen und ganzen zwar nur ein wenig, aber doch wahrnehmbar beeinträchtigt. Der Beginn der Phthaleinausscheidung bei Granulombildung war in 10 unter 19 Füllen etwas verspätet. Der Beginn der Phthaleinausscheidung nach intraveuöser Injektion des Farbstoffs betrug nümlich vor der Kieselgurinjektion $2^{\prime} 30^{\prime \prime}-7^{\prime}$, und $4^{\prime} 41^{\prime \prime}$ im Durchschnitt sümtlicher Fälle der Versuchsreihe A, während sich nach der Granulomentwicklung in der Niere der erste Farbstoff im Harn innerhalb $3^{\prime}-8^{\prime}$, durchschnittlich in $5^{\prime} 25^{\prime \prime}$, zeigte. Die ausgeschiedene Menge des Phenolsulfophthaleins in der 1 . Stunde machte $63,3-79,3 \%$, durchschnittlich $70,4 \%$, vor und 43,8-72,4\%, durchschnittlich 57,5\%, wach der Granulomentwicklung aus. In der 2 . Stunde dagegen betrug die ausgeschie- 
dene Phthaleinmenge 4,3-14,5\%, durchschnittlich 8,55\%, vor und 5,720,5\%, durchschnittlich 11,96\%, nach der Granulombildung. Die Farbstoffausscheidung in den weiteren Stunden war 3,4-12,5\%, durchschnittlich 6,06\%, vor und 5,2-12,7\%, durchschnittlich 7,92\%, nach der Granulombildung. Die Gesamtausscheidung des Phthaleins betrug demmach bei den Fällen der Versuchsreihe A 76,0-90,8\%, durchschnittlich 85,05\%, vor und 67,7-89,2\%, durchschnittlich $77,38 \%$, nach der Granulombildung.

Man konstatiert also bei den Versuchsfällen der Reihe A im Durchschnitte eine geringe Verspätung und Verminderung der Phthaleinausscheidung und dazu eine leichte Verschiebung der Ausscheidungskurve nach rechts. Die ausgeschiedenen Phthaleinmengen in den einzelnen Zeitabschnitten zeigten aber bei den Fïllen vor wie nach der Granulomentwicklung beinah dasselbe normale Verhalten ; der Kulminationspunkt der Ausscheidungskurve lag dabei in der 1. Stunde.

Bei den Fällen der Versuchsreihe B (siehe 'Tabelle II), wo Kicselgur in beide Nieren injiziert wurde, konnte man inbezug auf Gefrierpunkt und Harnstoffgehalt des Harns und Blutes, vor allem auch inbezug auf Phthaleinausscheidung aus den Nieren dasselbe wie bei der Versuchsreihe A konstatieren. Der Beginn der Phthaleinausscheidung war bei der Versuchsreihe $B$ auch nur etwas verspätet. Dieser trat $3^{\prime} 30^{\prime \prime}-6^{\prime}$, durchschnittlich $4^{\prime} 28^{\prime \prime}$, vor und $4^{\prime}-7^{\prime}$, durchschnittlich 5' 50", nach der Granulombildung ein. Die ausgeschiedene Menge des Farbstoffs betrug in der 1. Stunde $64,1-79,3 \%$, durchschnittlich $72,0 \%$, vor und $46,5-67,5 \%$, durchschnittlich $59,9 \%$, nach der Granulomentwicklung, während sie in der 2. Stunde 4,7-10,4\%, durchschnittlich 7,93\%, vor und 5,5-21,5\%, durchschnittlich 10,46\%, , nach der Granulombildung ausmachte. Die Ausscheidung des Farbstoffes in den weiteren Stunden war 3,3-7,1\%, durchschnittlich 4,85\%, vor und 1,5-12,5\%, durchschnittlich $7,43 \%$, nach der Granulombildung, so dass die Gesamtausscheidung des Phthaleins in der Versuchsreihe B $73,2-94,0 \%$, durchschnittlich $84,75 \%$, vor und $64,4-$ $87,1 \%$, durchschnittlich 77,74\%, nach der Granulombildung betrug.

Aus den Resultaten bemerkt man, dass auch bei den Versuchsfällen der Reihe B, wie bei denen der Versuchsreihe $A$, die Phthaleinausscheidung nach der Granulomentwicklung in den Nieren sowohl inbezug auf den Beginn wie auf Menge mehr oder weniger deutlich beeinträchtigt ist. Die Ausscheidungskurve des Phthaleins zeigt auch hier nach der Granulombildung in den Nieren durchschnittlich nur minimale Verschiebung nach rechts, ohne aber den Charakter der normalen Ausscheidungskurve 
zu verlieren. Ich konnte aber im Grad der durchschnittlichen Beeintrïchtigung der Phthaleinausscheidung keinen Unterschied zwischen den Fällen der Versuchsreihen A und B finden. Das könnte man daraus erklïren, dass bei der Versuchsreihe B trotz Granulombildung und dadurch verursachter Schüdigung des harnbereitenden Apparates in beiden Nieren das übrigbleibende Nierenparenchym kompensatorisch die ausgefallene Funktion übernimnt, was eine beinah normale Arbeitsleistung der Nieren, auch in Bezug auf die Farbstoffuusscheidung zur Folge hat.

\section{Schlussfolgerungen.}

1. Ich konnte durch Injektion einer sterilen Aufschwemmung fein zerriebener Kieselgur in das Nierenparenchym des Kaninchens an den Injektionsstellen mehr oder weniger deutliche Granulombildung hervorrufen. Ich habe dazu im ganzen 41 erwachsene Kaninchen verwendet, konnte aber nur bei 31 Tieren Bildung von Riesenzellengranulomen im Nierenparenchym feststellen. Bei 10 Versuchsfïllen, wo allzu dünne Kieselguraufschwemmung injiziert wurde, blieb Granulombildung aus.

2. Die Granulome kamen im Nierenparenchym als mehr oder minder grosse Kunötchen oder streifige oder nadelförmige Herde vor. Histologisch konnte man dabei, wie das Podwyssozki, Deton u. a. schon beschrieben haben, das typische Bild des sogenannten Riesenzellengranuloms konstatieren. Die Glomeruli und Harnkanälchen waren dabei innerhalb der Granulome nicht mehr als solche nachweisbar. An der Grenze zwischen dem Granulomgewebe und dem Nierenparenchym bemerkt man mehr oder minder deutliche Kapselbildung. In dem direkt an diese Bindegewebskapsel anstossenden, mehr oder minder von neugebildetem Bindegewebe durchwucherten Nierenparenchymteil konstatiert man Verminderung der Zahl der Glomeruli, teilweises Verschwinden der Harnkanälchen, Erweiterung des Bowmanschen Kapselraums und Erweiterung der Harnkanälchen und Zylinderbildung in ihren Lumen. Starke Tendenz zur Schrumpfung aber konnte man auch bei älteren Granulomknötchen nicht konstatieren.

3. In der Versuchsreihe A wurden bei 19 Kaninchen Granulome in der einen Niere erzeugt. Dabei konnte man nach der Granulomentwicklung nichts Besonderes hinsichtlich des spezifischen Gewichts des Harns, des Gefrierpunkts und des Harnstoffgehalts des Harns und des Blutes nachweisen. Die Phthaleinausscheidung war dabei durchschnittlich etwas verspïtet und vermindert, ohne dass aber der normale Verlauf der Ausscheidungskurve wesentlich verändert worden wäre. 
4. Bei der Versuchsreihe B, wo die Granulome in beiden Nieren erzeugt wurden, konnte man bei der Nierenfunktionsprüfung dasselbe Resultat wie bei der Versuchsreihe $A$ erheben. Dabei kam die funktionelle Beeinträchtigung der Nieren infolge mehrerer Granulombildungen in beiden Nieren kaum zum Ausdruck dank der Kompensation durch das noch gesund gebliebene Nierenparenchym.

\section{Literatur.}

(1) Bergel, S., Virchows Arch., 1921, 230, 461-47S.

(2) Deton, W., Ztschr. f. Krebsforsch., 1911, 10, 224-226.

(3) Herxheimer, G. u. F. Reinke, Lubarsch-Ostertags Ergebn. d. allg. Path. u. path. Anat., 1912, 16, II. Abt., 60-61.

(4) Konstantinowitsch, W., Virchows Arch., 1901, 163, 120-125.

(5) Ishikawa, M. u. M. Masugi, Transact. Japan. Pathol. Soc., 1922, 12, 302-307 (jap.) u. $86-87$ (dtsch.).

(6) Podwyssozki, W., Zieglers Beitr., 1910, 47, 270-281.

(7) Schirokogoroff, J. I., Virchow s Arch., 1911, 205, 166-169.

(8) Sugimura, Sh., Nihon Gekagakkai Zasshi (jap.), 1927, 28, 219-260.

(9) Sugimura, Sh. u. T. A om ura, Tohoku Journ. Exper. Med., 1926, 7, 125-143. 